\title{
Prognostic Significance of Neutrophil-to- Lymphocyte Ratio in Ovarian Cancer: A Systematic Review and Meta-Analysis of Observational Studies
}

\author{
Qi-tao Huang ${ }^{\mathrm{a}}$ Lin Zhou ${ }^{\mathrm{b}}$ Wen-juan Zeng ${ }^{\mathrm{a}}$ Qian-qian Ma ${ }^{\mathrm{b}}$ Wei Wang ${ }^{\mathrm{a}}$ \\ Mei Zhong ${ }^{a}$ Yan-hong Yu ${ }^{a}$ \\ aDivision of Obstetrics and Gynecology, Nanfang Hospital, Southern Medical University, Guangzhou, \\ bSouthern Medical University, Guangzhou, China
}

\section{Key Words}

Ovarian cancer $\cdot$ Inflammatory $\cdot$ Neutrophil $\cdot$ Lymphocyte $\bullet$ Prognosis

\begin{abstract}
Background and Aims: Published data on the prognostic role of neutrophil-to-lymphocyte ratio (NLR) in ovarian cancer are controversial. We conducted this meta-analysis to obtain a more accurate assessment of prognostic significance of NLR in ovarian cancer. Materials and Methods: We conducted a systematic literature search using the electronic databases PubMed, Web of Science, and Embase up to May 2016. Hazard ratio (HR) and odd ratio (OR) with $95 \%$ confidence interval $(95 \%$ CI) were calculated. Subgroup analyses were carried out to explore the source of heterogeneity. Statistical analysis was performed using Stata 10.0. Results: A total of 12 studies, consisting of 3,854 patients, which met our criterion were selected in this meta-analysis. Our pooled results showed that high pre-treatment NLR level was significantly associated with poorer overall survival (OS) (HR: 1.69, 95\% CI 1.29-2.22) and shorter progression free survival (PFS) (HR 1.63, 95\% CI 1.27-2.09). Additionally, increased NLR was also significantly correlated with advanced FIGO stage (OR 2.32, 95\% CI1.79-3.00), higher serum level of CA-125 (OR 3.33, 95\% CI 2.43-4.58), more extensive ascites (OR 3.54, $95 \%$ CI 2.31-5.42) as well as less chemotheraputic response (OR $0.53,95 \%$ CI $0.40-0.70$ ). The findings from most of subgroup meta-analyses were consistent with those from the overall meta-analyses. Conclusions: Elevated pre-treatment NLR could served as a predicative factor of poor prognosis for ovarian cancer patients

\section{Introduction}

Ovarian cancer is one of the most common and lethal female malignancy, and there are over 200,000 estimated new cases and 150,000 deaths every year globally [1,2]. After

Q.-T. Huang and L. Zhou contributed equally to this work.

Dr. Qi-tao Huang

or Mei Zhong, Professor of Obstetrics

and Gynecology
1838 North Guangzhou Avenue, Guangzhou 510515 (People's Republic of China) E-Mail 15920454515@163.com / zhongm1960@163.com 
primary therapy including debulking surgery and platinum-based adjuvant chemotherapy, approximately half of the patients will relapse within 1 year, and the five-year overall survival rate is less than 50\% [3-5]. Therefore, effective bio-markers for individualized prediction of therapy outcomes and prognosis are urgently warranted.

The associations between inflammation and tumor development have gained much interest in the past few decades [6]. Neutrophil to lymphocyte ratio (NLR), defined as the ratio of neutrophil to lymphocyte count, is a marker for evaluating the systemic potential balance between neutrophil-dependent pro-tumor inflammation and lymphocyte-associated anti-tumor immune response [7]. A higher level of NLR could represent a trend towards increased pro-tumor inflammation and decreased anti-tumor immune capacity.

Accumulating evidence demonstrates that NLR has prognostic significance in patients with various types of cancers [8-11], including gastrointestinal tract malignancies, pancreatic carcinoma, hepatocellular cancer, non-small-cell lung cancer as well as cervical carcinoma. Recently, several studies evaluated the prognostic significance of NLR in patients with ovarian cancer [12-25]. But the results of each individual studies were inconsistent. Therefore, the prognostic significance of NLR in ovarian cancer remained controversial. To clarify this issue, we performed this systematic review and meta-analysis to obtain a more reliable assessment of prognostic significance of NLR in patients with ovarian cancer.

\section{Materials and Methods}

This systematic review and meta-analysis was performed following the guidance provided in the Cochrane Handbook and was reported according to the Meta-analysis of Observational Studies in Epidemiology (MOOSE) guidelines [26].

\section{Search strategy}

We conducted a systematic literature search using the electronic databases PubMed, Web of Science, and Embase up to May 2016. Search terms included "neutrophil to lymphocyte ratio", "NLR", "ovarian" and "tumor, cancer, neoplasm, carcinoma or malignancy". The titles and abstracts of potential references were scanned carefully to exclude irrelevant articles. The remaining studies were assessed to identify the topic of interest, and full texts were then reviewed comprehensively.

\section{Selection criteria}

A study was included if it met the following criteria: (1) included patients with ovarian cancer diagnosed histopathologically; (2) provided pre-treatment and/or post-treatment NLR and cut-off values, (3) evaluated the associations between pre-treatment and/or post-treatment NLR and survival outcomes. Exclusion criteria were (1) review articles, editorial comments, letters, expert opinion, conference abstracts, or case reports; (2) insufficient data for estimating hazard ratios (HRs) and 95\% confidence intervals (CIs); or (3) full text unavailable and non-English article.

All assessments were conducted independently by two reviewers to assure accuracy of inclusive studies. Multivariate data were preferred to univariate data if both were provided. However, univariate data were acceptable if no multivariate results were presented.

\section{Data extraction}

Two investigators independently gathered information from each eligible study. Data was extracted as follows: surname of first author, study country, year of publication, sample size, cancer stage, treatment method, cut-off value defining elevated NLR and hazard ratios (HRs) with 95\% confidence intervals (CIs) for overall survival (OS) and progress-free survival (PFS)/recurrence-free survival (RFS). Disagreements in data extraction were resolved through discussion.

Assessment of methodological quality

Two independent investigators assessed the quality of each study included using the NewcastleOttawa Quality Assessment Scale (NOS) [27]. On a score scale from 0 to 9, a study with 7 or more stars was considered as high-quality. 


\section{Statistical analysis}

The HR and and corresponding 95\% CI were used to evaluate the prognostic efficiency of NLR on ovarian cancer. HRs and 95\% CIs, either obtained directly from each articles or calculated from indirect data, were synthesized as the effective value. In addition, the relationship between NLR and clinical-pathological features were reported as odd ratios (ORs) and 95\% CIs. Cochran's Q test and Higgins I-squared statistic were adopted to test the heterogeneity of pooled data. $I^{2}<50 \%$ and $p>0.1$ indicated no significant heterogeneity and fixed-effects model was applied to combine the effective value [28]. Otherwise, a random-effects model was adopted. All statistical analyses were performed using STATA 12.0 software (StataCorp LP, TX, USA).

Subgroup analyses were performed to investigate the associations of NLR with clinical features in relation to geographic area, statistical methods, sample size, cancer stage, lymph node involvement, NLR cutoff value, and follow-up duration. Moreover, a sensitivity analysis was performed to examine the robustness of the pooled results. We performed summary receiver operating characteristic to determine the NLR cutoff values as described [29,30]. We used Youden index to further confirm our results [31].

\section{Results}

Selection and characteristics of included eligible studies

A flowchart for the selection of eligible studies is demonstrated in Fig. 1. A total of 359 studies was retrieved and screened by title and abstract. 296 studies were excluded after the initial assessment of title and abstract. Among the remaining 63 articles, 36 were further excluded because they were letters, comments, editorials, or reviews. The full texts of the remaining 27 articles were evaluated. A total of 13 full-text articles were excluded, including 9 without available data and 4 without NLR category.

The basic information of the selected studies was summarized in Table 1.

\section{Association of pre-treatment NLR with overall survival}

The association between NLR and OS was assessed in 12 studies consisting of 3,854 patients. The pooled estimate indicated that a significantly shorter OS in ovarian cancer patients with high NLR compared to those with low NLR (HR: 1.69, 95\% CI 1.29-2.22) (Fig. 2). Because the heterogeneity test showed that significant heterogeneity $\left(\mathrm{I}^{2}=68.3 \%, \mathrm{P}<0.001\right)$ exists between the studies, a random-effects model was used for the analysis.

Association of pre-treatment NLR with progression free survival

The association between NLR and PFS was evaluated in 5 studies including 2,071 patients. Those with high pre-treatment NLR had a significantly poorer PFS than those with low NLR (HR 1.63, 95\% CI 1.27-2.09) (Fig. 3). Because significant heterogeneity was observed among these studies $\left(\mathrm{I}^{2}=56.6 \%, \mathrm{P}=0.024\right)$, a random-effects model was used for the analysis.

Correlations between NLR and clinical-pathological features

The correlations between NLR and the clinical characteristics such as tumor grade of differentiation, serum level of CA-125, ascites, residual tumor size, FIGO stage, lymph node
Fig. 1. Flow diagram of the study selection process.

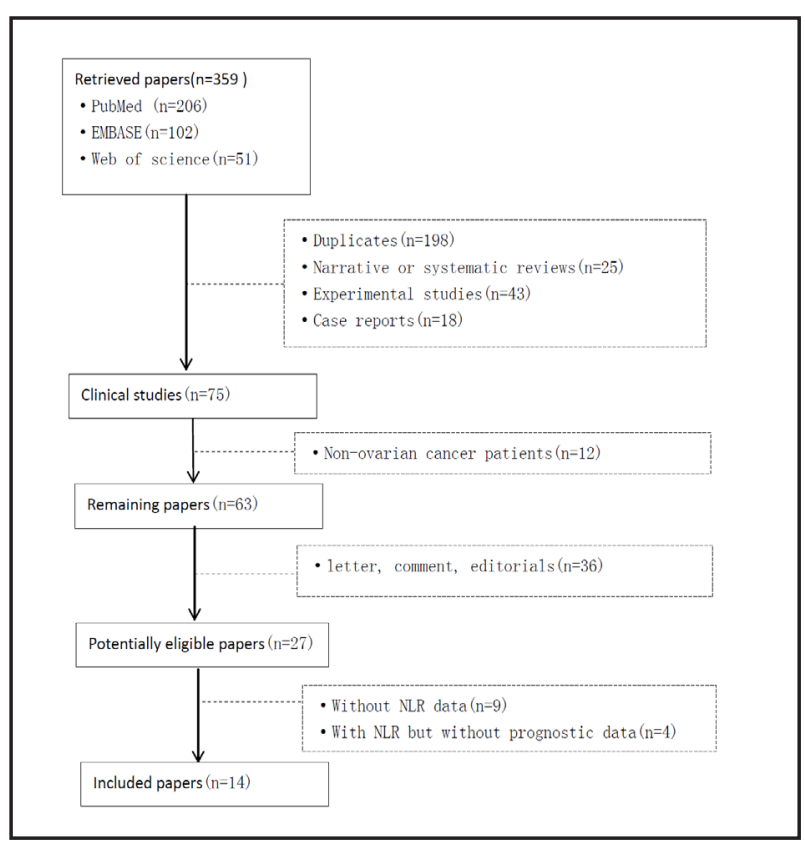


Table 1. Characteristics of all identified studies. HR: hazard ratio;CI: confidence interval; OS: overall survival;PFS: progression-free survival; DFS: disease-free survival;"-": not reported; EOC: epithelial ovarian cancer ;OCCC: ovarian clear cell carcinoma; SOC: serous ovarian cancer; NEOC: non-epithelial ovarian cancer; HGSC: high-grade serous ovarian cancer; FIGO: International Federation of Gynecology and Obstetrics;Grade; 2.FIGO stage; 3.Ascites; 4.CA-125; 5.Cytoreductive; 6.Chemosensitivity; 7.Lymph node metastasis.The study performed by Wang et al. [17]contained different levels of NLR. \# $1.86<\mathrm{NLR} \leq 2.64$; $\# \# N L R>3.77$

\begin{tabular}{|c|c|c|c|c|c|c|c|c|c|}
\hline Study & Year Country & Duration & Follow-up (m) & \multicolumn{3}{|c|}{ Multivariate analysis-HR(95\%CI) } & $\begin{array}{l}\text { Univariate } \\
\text { analysis-HR(95\%CI })\end{array}$ & $\operatorname{Age}(y)$ & $\begin{array}{l}\text { Histologic } \\
\text { type }\end{array}$ \\
\hline Cho H [12] & 2009 Korea & \multicolumn{2}{|c|}{$2003-200620.9$} & \multicolumn{3}{|l|}{ OS:8.42 (1.09-64.84) } & OS:6.05 (1.77-20.71) & - & EOC \\
\hline $\begin{array}{l}\text { Thavaramara T } \\
\text { [13] }\end{array}$ & 2011 Thailand & \multicolumn{2}{|c|}{$2004-200960$} & \multicolumn{3}{|c|}{$\begin{array}{l}\text { OS:preoperative } 1.43(0.625-3.33) \\
\text { postoperative } 0.714(0.303-1.429) \\
\text { PFS:preoperative1.43(0.71-3.33) } \\
\text { postoperative } 0.909(0.476-1.667)\end{array}$} & - & $49.6(37.1-61.1)$ & EOC \\
\hline Asher V [14] & 2011 UK & \multicolumn{2}{|c|}{$1988-199824.5$} & \multicolumn{3}{|l|}{ OS:0.865(0.521-1.437) } & & $62(24-90)$ & EOC \\
\hline Kim HS [15] & 2016 Korea & \multicolumn{2}{|c|}{$1997-201246[62.1-192.9]$} & \multicolumn{3}{|l|}{ PFS:2.04 (1.01-4.00) } & $\begin{array}{l}\text { OS:3.45(1.47-7.70) } \\
\text { PFS:2.94(1.50-5.60) }\end{array}$ & $53(30-86)$ & OCCC \\
\hline Williams KA [16] & \multirow[t]{2}{*}{2014 American } & \multicolumn{2}{|c|}{ n 1992-2013 68.4(1-252) } & \multicolumn{3}{|l|}{ OS:1.43 (1.13-1.81) } & - & 55 & EOC \\
\hline Wang Y [17] & & 2009-201 & $043(33-70.4)$ & \multicolumn{3}{|c|}{$\begin{array}{l}\text { OS\#:1.877 (0.622-5.324) } \\
\text { OS\#\#:8.567 (2.808-26.136) } \\
\text { PFS:3.554 (1.389-9.096) }\end{array}$} & $\begin{array}{l}\text { OS\#:2.403 (1.104-6.409) } \\
\text { OS\#\#:6.866 (2.797-16.851) } \\
\text { PFS:3.191 (1.514-6.728) }\end{array}$ & 50 & SOC \\
\hline Zhang WW [18] & 2015 China & $2000-201$ & $243(2-164)$ & 一 & & & $\begin{array}{l}\text { OS:2.172 (1.545-3.054) } \\
\text { PFS:2.012 (1.476-2.741) }\end{array}$ & $50.6 \pm 11.1(24-76)$ & EOC \\
\hline Nakamura K [19] & 2016 Japan & $2005-201$ & & $\begin{array}{l}\text { mortality within } 100 \text { day } \\
14.128(1.207-165.400)\end{array}$ & & & $\begin{array}{l}\text { mortality within } 100 \text { days: } \\
31.667(3.887-257.963)\end{array}$ & $54.9(33-78)$ & EOC \\
\hline Wang YQ [20] & 2016 China & 2006-201 & 3116 & OS:3.37(1.39-8.15) PFS & $0(1.03-$ & $-4.70]$ & $\begin{array}{l}\text { OS:2.90 (1.66-5.05) } \\
\text { PFS:2.11 (1.29-3.46) }\end{array}$ & $52.27(38.18-66.36]$ & EOC/NEOC \\
\hline Feng Z [21] & 2016 China & $2005-201$ & $329(1-115)$ & $\begin{array}{l}\text { OS:1.189 (0.940-1.504) } \\
(1.052-1.484)\end{array}$ & & PFS: 1.250 & - & $56(30-90)$ & HGSC \\
\hline Miao Y [22] & 2016 China & $2005-201$ & $072(61-97)$ & $\begin{array}{l}\text { OS: } 1.616(1.138-2.297) \\
(1.225-2.453)\end{array}$ & & PFS: 1.733 & $\begin{array}{l}\text { OS: } 4.970(3.794-6.511) \\
\text { PFS: } 5.093(3.886-6.676)\end{array}$ & $55(45-84)$ & EOC \\
\hline $\begin{array}{l}\text { Raungkaewmanee } \\
\text { S [23] }\end{array}$ & 2012 Thailand & $2004-201$ & $028.3(6.1-94.4)$ & $\begin{array}{l}\text { OS:0.85 }(0.46-1.60) \\
\text { PFS:0.89 }(0.48-1.64)\end{array}$ & & & - & $53(23-85)$ & EOC \\
\hline Yildirim MA [24] & 2014 Sultan & 2006-201 & & 一 & & & - & 43 & EOC/NEOC \\
\hline Bakacak M [25] & 2016 Turkey & $2007-201$ & $5-$ & 一 & & & 一 & 53 & EOC/NEOC \\
\hline Study & Patient cl & linical pare & ameters Sample S & Sampling time & ff Stage & End & Treatme & Study & $\mathrm{y}$-design NOs \\
\hline Cho H [12] & 一 & & 999 & pretreatment & I-IV & $\mathrm{OS} / \mathrm{DF}$ & surgery & retros & spective 8 \\
\hline Thavaramara T [13] & 2,5 & & 128 & pre/postoperative 2.6 & advan & nced OS/ PF & surgery/ & chemotherapy retros & spective 7 \\
\hline Asher V [14] & - & & 235 & preoperative & I-IV & OS & surgery/ & chemotherapy retros & spective 7 \\
\hline Kim HS [15] & $2,5,6$ & & 109 & postoperative & I-IV & PFS & surgery/ & chemotherapy retros & spective 8 \\
\hline Williams KA [16] & $1,2,3,5$ & & 519 & pretreatment & I-IV & OS & surgery & retros & spective 8 \\
\hline Wang Y [17] & $1,2,3,4,5,6$ & 6,7 & 126 & preoperative & I-IV & $\mathrm{OS} / \mathrm{PF}$ & surgery/ & chemotherapy retros & spective 8 \\
\hline Zhang WW [18] & - & & 190 & preoperative & I-IV & $\mathrm{OS} / \mathrm{PF}$ & surgery/ & chemotherapy retros & spective 6 \\
\hline Nakamura K [19] & - & & 30 & pretreatment & advan & nced morta & lity within 100 days surgery/ & chemotherapy retros & spective 7 \\
\hline Wang YQ [20] & $2,4,7$ & & 143 & preoperative & I-IV & $\mathrm{OS} / \mathrm{PF}$ & surgery & retros & spective 7 \\
\hline Feng Z [21] & $2,3,4,5,6$ & & 875 & preoperative & I-IV & $\mathrm{OS} / \mathrm{PF}$ & surgery & retros & spective 8 \\
\hline Miao Y [22] & 6 & & 334 & pretreatment & I-IV & $\mathrm{OS} / \mathrm{PI}$ & surgery/ & chemotherapy retros & spective 8 \\
\hline Raungkaewmanee S & $S[23] 1,2,3$ & & 166 & pretreatment & I-IV & $\mathrm{OS} / \mathrm{PL}$ & surgery & retros & spective 6 \\
\hline Yildirim MA [24] & 2 & & 316 & pre/postoperative 2.11 & I-IV & OS & surgery & retros & spective 5 \\
\hline Bakacak M [25] & 2 & & 218 & preoperative & - & 一 & surgery & retros & spective 7 \\
\hline
\end{tabular}

involvement, chemotherapeutic response are presented in Table 2. Increased pre-treatment NLR was significantly correlated with advanced FIGO stage (OR 2.32, 95\% CI1.79-3.00), higher serum level of CA-125 (OR 3.33, 95\% CI 2.43-4.58), more extensive ascites (OR $3.54,95 \%$ CI 2.31-5.42) as well as less chemotheraputic response (OR 0.53, $95 \%$ CI $0.40-0.70$ ). Four, two and one studies were available for the pooled analysis with regard to residual tumor size, lymph node metastasis and tumor grade of differentiation. No significant correlations between NLR with the abo-

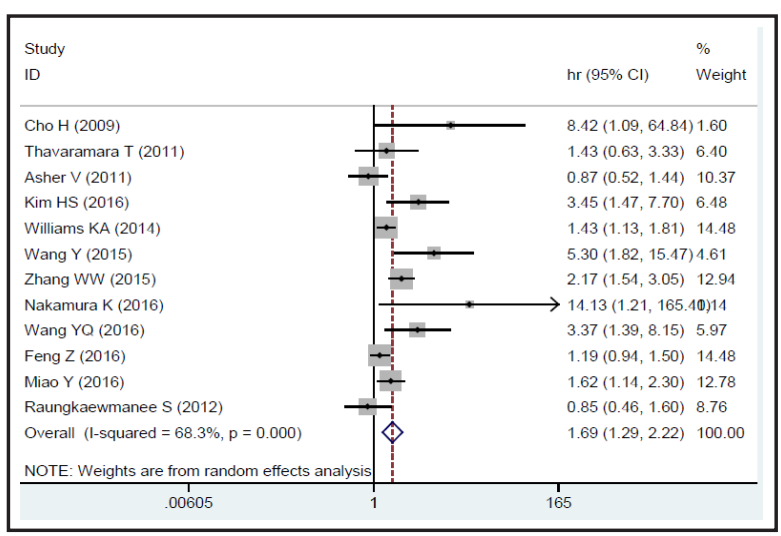

Fig. 2. Forest plot of the correlation between NLR and OS in ovarian cancer patients. 
ve-mentioned clinical-pathological features were observed.

\section{Subgroup analysis}

Subgroup analyses are conducted to explore the possible sources of heterogeneity. Results of subgroup meta-analyses are summarized in Table 3 .

\section{Publication bias analysis}

The funnel plots showed a low probability of publication bias (Fig. 3 A\&B). Consistently, the Egger's and Begger's regression tests demonstrated little evidence of publication bias for OS $(\mathrm{P}=0.061 ; \mathrm{P}=0.150)$ and for PFS ( $\mathrm{P}=0.203 ; \mathrm{P}=0.536)$, respectively.

\section{Sensitivity analysis}

To assess the stability of every pooled result in our meta-analysis, we performed a sensitivity analysis for every analysis by sequential omission of the individual study. The pooled HRs for OS and PFS were not significantly changed, which suggested the robustness of the results.

\section{Discussion}

Accumulating evidence demonstrates that inflammation exerts an essential role in cancer formation, development and progression through facilitating angiogenesis, proliferation and preventing tumors from apoptosis [7]. Previous studies demonstrate that hematological inflammatory markers (including C-reactive protein, albumin, neutrophils and so on) could help predicting survival in patients with various types of cancers [12-15]. Among these predictors, NLR is a reproducible and widely available laboratory hematological marker in our routine clinical practice. Neutrophils have been considered to be the primary

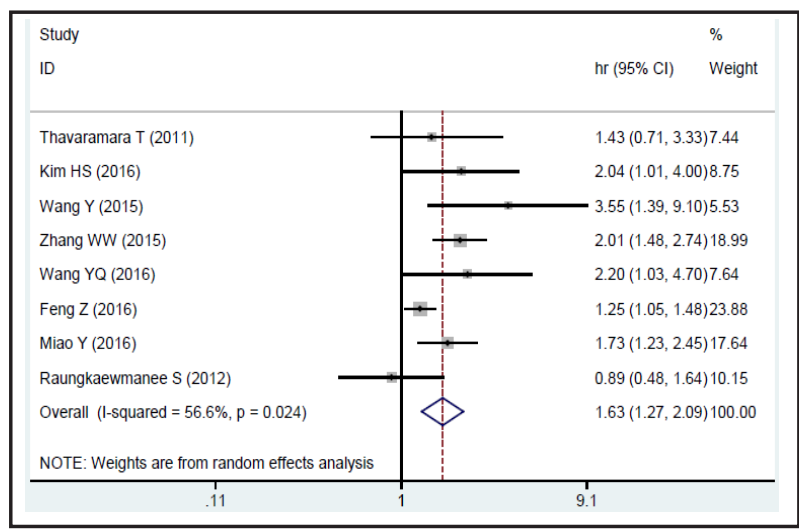

Fig. 3. Forest plot of the correlation between NLR and PFS in ovarian cancer patients.

Table 2. Correlation between high NLR and clinical parameters

\begin{tabular}{lccccc}
\hline Clinical parameters & N & OR & LCI & HCI & $I^{2}$ \\
\hline FIGO stage & 7 & 2.32 & 1.79 & 3.00 & $41.4 \%$ \\
CA-125 & 3 & 3.33 & 2.43 & 4.58 & $0.0 \%$ \\
Ascites & 2 & 3.54 & 2.31 & 5.42 & $40.2 \%$ \\
Chemosensitivity & 3 & 0.53 & 0.40 & 0.70 & $22.7 \%$ \\
Residual tumor size & 4 & 1.70 & 0.93 & 3.10 & $51.7 \%$ \\
Lymph node metastasis & 2 & 0.86 & 0.08 & 9.20 & $94.2 \%$ \\
Tumor grade of differentiation & 1 & 1.21 & 0.60 & 2.44 & $0.0 \%$ \\
\hline
\end{tabular}

Table 3. Result of the meta-analysis on predictive value of NLR in ovarian cancer patients. *Six studies $[12,14,17$, $19,20,22]$ analyzed correlation between NLR and overall survival using both univariate and multivariate analysis. Four studies [15, 17, 20, 22] analyzed correlation between NLR and disease-free survival using both univariate and multivariate analysis. \#The study performed by Wang et al. [17] contained different levels of NLR. For overall survival: $1.86<\mathrm{NLR} \leq 2.64, \mathrm{HR} 1.877,95 \% \mathrm{CI}(0.662-5.324)$; NL$\mathrm{R}>3.77$,HR 8.567, 95\% CI (2.808-26.136)

\begin{tabular}{|c|c|c|c|c|c|c|c|c|}
\hline & \multicolumn{4}{|c|}{ Overall survival } & \multicolumn{4}{|c|}{ Progression free survival } \\
\hline & $\mathrm{N}$ & HR & LCI & $\mathrm{HCI}$ & $\mathrm{N}$ & HR & LCI & $\mathrm{HCI}$ \\
\hline Overall & 12 & 1.69 & 1.29 & 2.22 & 8 & 1.63 & 1.27 & 2.09 \\
\hline \multicolumn{9}{|c|}{ Geographic area } \\
\hline 1.Asian & 10 & 1.98 & 1.39 & 2.82 & 8 & 1.63 & 1.27 & 2.09 \\
\hline 2.non-Asian & 2 & 1.17 & 0.72 & 1.90 & 0 & - & - & - \\
\hline \multicolumn{9}{|c|}{ Statistical methods* } \\
\hline 1.Univariate & 8 & 3.33 & 2.15 & 5.15 & 5 & 2.91 & 1.82 & 4.46 \\
\hline 2.Multivariate & 10 & 1.51 & 1.14 & 2.01 & 7 & 1.38 & 1.20 & 1.59 \\
\hline \multicolumn{9}{|l|}{ Sample size } \\
\hline $1 .<300$ & 8 & 2.01 & 1.23 & 3.29 & 6 & 1.83 & 1.46 & 2.29 \\
\hline $2 . \geq 300$ & 4 & 1.37 & 1.18 & 1.59 & 2 & 1.42 & 1.04 & 1.94 \\
\hline \multicolumn{9}{|l|}{ NLR standard } \\
\hline $1 .<3$ & 5 & 1.85 & 0.95 & 3.58 & 4 & 1.64 & 0.94 & 2.86 \\
\hline $2 . \geq 3$ & $8^{\#}$ & 1.74 & 1.27 & 2.39 & 4 & 1.65 & 1.32 & 2.21 \\
\hline \multicolumn{9}{|l|}{ Follow-up } \\
\hline 1. $<60$ & 8 & 1.85 & 1.17 & 2.92 & 5 & 1.61 & 1.12 & 2.31 \\
\hline $2 . \geq 60$ & 4 & 1.54 & 1.28 & 1.85 & 3 & 1.75 & 1.30 & 2.34 \\
\hline
\end{tabular}

source of circulating VEGF, which play a critical role in tumor-associated angiogenesis [1315]. In addition, neutrophils could enhance the producing of many inflammatory cytokines such as tumor necrosis factor, interleukin 1 , interleukin 6 , and therefore provide a favorable 
micro-environment for tumor survival and proliferation [8]. Conversely, lymphocytes exert a critical role in cancer-specific immune response [32]. It has been shown that the increased infiltration of lymphocytes in tumor tissue is associated with good prognosis [33].

In the present study, we aimed to investigate the prognostic value of NLR in patients with ovarian cancer. Our results indicated that an elevated NLR was associated with poorer OS and shorter PFS in ovarian cancer patients, which was in accordance with the results from studies with several other cancer types.

Previous studies reported that NLR level was significantly associated with patient clinical characteristics [34, 35]. For instance, Yodying et al. [34] evaluated the prognostic role of NLR in esophageal cancer and indicated that NLR was associated with tumor invasion and lymph node metastasis. Xue et al. [35] observed that NLR was associated with vascular invasion in hepatic carcinoma. In this study, we also explored the correlations between NLR and clinical-pathological features in ovarian cancer patients. Our results showed that higher NLR was positively correlated with an advanced FIGO stage, an increased CA125 level and more extensive ascites in ovarian cancer patients. Therefore, we proposed that NLR might reflect ovarian cancer burden and inflammatory severity and significantly affect intrinsic tumor characteristics in patients with ovarian cancer. Moreover, our pooled results suggested that greater proportions of the ovarian cancer patients with chemo-resistance had higher NLR levels compared with those of chemo-sensitive, which suggested the potential application of NLR to predict chemotherapy response in ovarian cancer patients. Our meta-analysis also investigated the association between NLR level and tumor grade of differentiation, residual tumor size after cytoreduction and lymph node involvement. However, since limited studies were available, we still cannot draw a robust conclusion currently.

There were several limitations should be clarified in this study. First, most of the studies selected in this meta-analysis were retrospective, observational studies, and no prospective cohort study was identified. Therefore, it may be more susceptible to bias in data analysis. Second, a previous systemic review demonstrated that increased NLR predicted poor PFS with prostate cancer only in Asians, but not in Caucasians, which could be attributed to the ethnicity heterogeneity [36]. In this meta-analysis, majority of included articles came from Asian countries. Therefore, our current conclusions may be not suitable to be applied to other populations. Third, heterogeneity was observed in this meta-analysis. This heterogeneity may be partially caused by geographic area, statistical methods, sample size, NLR cut-off value, and the follow-up duration. For instance, studies with significant results are easier to be published than those with null or insignificant results. Thus the pooled HR may be potentially overestimated. Moreover, dichotomized cut-off values of NLR differed significantly among the studies. The further subgroup analyses showed that a pre-treatment NLR $\geq 3$ was significantly associated with poorer overall survival, and the predictive value were not significant when NLR $<3$. Thus, a higher NLR cut-off may increase the specificity for predicting a poor prognosis. However, significant heterogeneity was observed in these subgroups, and a random-effect model was employed to produce more conservative results. In the future, more prospective, original research is required to determine the most suitable cut-off value of NLR in ovarian cancer patients

Despite the limitations, our meta-analysis also has some strengths. To the best of our knowledge, this meta-analysis is the first to evaluate the prognostic role of a pre-treatment peripheral blood NLR in ovarian cancer. Moreover, our results showed that a significantly positive correlations between NLR and the clinical features of ovarian cancer, such as advanced FIGO stage, more extensive ascites as well as less chemotheraputic response. Thus, NLR could have a wider clinical application regarding the prognostic assessment of ovarian cancer and might be useful in stratifying patients and in determining individual treatment plans in the future.

In conclusion, our meta-analysis of currently available clinical evidence demonstrates that a high pre-treatment NLR is associated with a poor prognosis in ovarian cancer patients. NLR could serve as a promising prognostic marker because it is available from blood routine test in daily clinical practice, which are convenient, low cost, and reproducible. In addition, 
considering that NLR level is associated with several pathological features of ovarian cancer including tumor staging, ascites and chemotherapy response rates, it would be interesting to explore whether decreasing the inflammatory conditions, such as lowing NLR level could serve as an adjuvant therapy and prolong the survival of ovarian cancer patients in the near future.

\section{Funding}

This work was supported by the National Natural Science Foundation of China (81401208, 81671466), Natural Science Foundation of Guangdong Province (2015A030310025), the President Grant from Nanfang Hospital (2014B003, 2016B019) and National Undergraduate Training Program for Innovation and Entrepreneurship (201612121007).

\section{Disclosure Statement}

The authors have no competing interests to declare.

\section{References}

Jayson GC, Kohn EC, Kitchener HC, Ledermann JA: Ovarian cancer. Lancet 2014;384:1376-1388.

Clarke-Pearson DL: Clinical practice. Screening for ovarian cancer. N Engl J Med 2009;361:170-177.

Li Y, Li Y, Zhang J, Zheng C, Zhu H, Yu H, Fan L: Circulating Insulin-Like growth factor-1 level and ovarian cancer risk. Cell Physiol Biochem 2016;38:589-597.

4 Tangjitgamol S, Manusirivithaya S, Laopaiboon M, Lumbiganon P, Bryant A: Interval debulking surgery for advanced epithelial ovarian cancer. Cochrane Database Syst Rev 2010:D6014.

-5 Vergote I, Trope CG, Amant F, Kristensen GB, Ehlen T, Johnson N, Verheijen RH, van der Burg ME, Lacave AJ, Panici PB, Kenter GG, Casado A, Mendiola C, Coens C, Verleye L, Stuart GC, Pecorelli S, Reed NS: Neoadjuvant chemotherapy or primary surgery in stage IIIC or IV ovarian cancer. N Engl J Med 2010;363:943-953.

6 Idzko M, Ferrari D, Eltzschig HK: Nucleotide signalling during inflammation. Nature 2014;509:310-317.

7 Moses K, Brandau S: Human neutrophils: Their role in cancer and relation to myeloid-derived suppressor cells. Semin Immunol 2016;28:187-196.

8 Grivennikov SI, Greten FR, Karin M: Immunity, inflammation, and cancer. Cell 2010;140:883-899.

-9 Templeton AJ, McNamara MG, Seruga B, Vera-Badillo FE, Aneja P, Ocana A, Leibowitz-Amit R, Sonpavde G, Knox JJ, Tran B, Tannock IF, Amir E: Prognostic role of neutrophil-to-lymphocyte ratio in solid tumors: A systematic review and meta-analysis. J Natl Cancer Inst 2014;106:u124.

10 Malietzis G, Giacometti M, Kennedy RH, Athanasiou T, Aziz O, Jenkins JT: The emerging role of neutrophil to lymphocyte ratio in determining colorectal cancer treatment outcomes: A systematic review and metaanalysis. Ann Surg Oncol 2014;21:3938-3946.

11 Huang QT, Man QQ, Hu J, Yang YL, Zhang YM, Wang W, Zhong M, Yu YH: Prognostic significance of neutrophil-to-lymphocyte ratio in cervical cancer: A systematic review and meta-analysis of observational studies. Oncotarget Doi:10.18632/oncotarget.15157.

12 Cho H, Hur HW, Kim SW, Kim SH, Kim JH, Kim YT, Lee K: Pre-treatment neutrophil to lymphocyte ratio is elevated in epithelial ovarian cancer and predicts survival after treatment. Cancer Immunol Immunother 2009;58:15-23.

13 Thavaramara T, Phaloprakarn C, Tangjitgamol S, Manusirivithaya S: Role of neutrophil to lymphocyte ratio as a prognostic indicator for epithelial ovarian cancer. J Med Assoc Thai 2011;94:871-877.

14 Asher V, Lee J, Innamaa A, Bali A: Preoperative platelet lymphocyte ratio as an independent prognostic marker in ovarian cancer. Clin Transl Oncol 2011;13:499-503.

15 Kim HS, Choi HY, Lee M, Suh DH, Kim K, No JH, Chung HH, Kim YB, Song YS: Systemic inflammatory response markers and CA-125 levels in ovarian clear cell carcinoma: A two center cohort study. Cancer Res Treat 2016;48:250-258.

-16 Williams KA, Labidi-Galy SI, Terry KL, Vitonis AF, Welch WR, Goodman A, Cramer DW: Prognostic significance and predictors of the neutrophil-to-lymphocyte ratio in ovarian cancer. Gynecol Oncol 2014;132:542-550. 


\section{Cellular Physiology Cell Physiol Biochem 2017;41:2411-2418

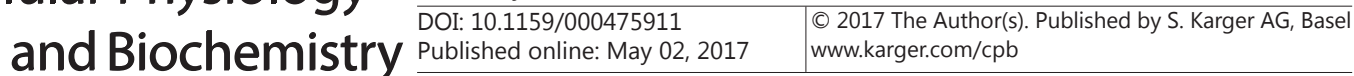

Huang et al.: NLR and Ovarian Cancer

17 Wang Y, Liu P, Xu Y, Zhang W, Tong L, Guo Z, Ni H: Preoperative neutrophil-to-lymphocyte ratio predicts response to first-line platinum-based chemotherapy and prognosis in serous ovarian cancer. Cancer Chemother Pharmacol 2015;75:255-262.

-18 Zhang WW, Liu KJ, Hu GL, Liang WJ: Preoperative platelet/lymphocyte ratio is a superior prognostic factor compared to other systemic inflammatory response markers in ovarian cancer patients. Tumour Biol 2015;36:8831-8837.

19 Nakamura K, Nagasaka T, Nishida T, Haruma T, Ogawa C, Kusumoto T, Seki N, Hiramatsu Y: Neutrophil to lymphocyte ratio in the pre-treatment phase of final-line chemotherapy predicts the outcome of patients with recurrent ovarian cancer. Oncol Lett 2016;11:3975-3981.

20 Wang YQ Jin C, Zheng HM, Zhou K, Shi BB, Zhang Q, Zheng FY, Lin F: A novel prognostic inflammation score predicts outcomes in patients with ovarian cancer. Clin Chim Acta 2016;456:163-169.

-21 Feng Z, Wen H, Bi R, Ju X, Chen X, Yang W, Wu X: Preoperative Neutrophil-to-Lymphocyte ratio as a predictive and prognostic factor for High-Grade serous ovarian cancer. Plos One 2016;11:e156101.

22 Miao Y, Yan Q, Li S, Li B, Feng Y: Neutrophil to lymphocyte ratio and platelet to lymphocyte ratio are predictive of chemotherapeutic response and prognosis in epithelial ovarian cancer patients treated with platinum-based chemotherapy. Cancer Biomark 2016;17:33-40.

23 Raungkaewmanee S, Tangjitgamol S, Manusirivithaya S, Srijaipracharoen S, Thavaramara T: Platelet to lymphocyte ratio as a prognostic factor for epithelial ovarian cancer. J Gynecol Oncol 2012;23:265-273.

-24 Yildirim MA, Seckin KD, Togrul C, Baser E, Karsli MF, Gungor T, Gulerman HC: Roles of neutrophil/ lymphocyte and platelet/lymphocyte ratios in the early diagnosis of malignant ovarian masses. Asian Pac J Cancer Prev 2014;15:6881-6885.

25 Bakacak M, Serin S, Ercan O, Kostu B, Bostanci MS, Bakacak Z, Kiran H, Kiran G: Utility of preoperative neutrophil-to-lymphocyte and platelet-to-lymphocyte ratios to distinguish malignant from benign ovarian masses. J Turk Ger Gynecol Assoc 2016;17:21-25.

-26 Stroup DF, Berlin JA, Morton SC, Olkin I, Williamson GD, Rennie D, Moher D, Becker BJ, Sipe TA, Thacker SB: Meta-analysis of observational studies in epidemiology: A proposal for reporting. Meta-analysis of Observational Studies in Epidemiology (MOOSE) group. JAMA 2000;283:2008-2012.

27 Wells G, Shea BO, Connell D: The Newcastle-Ottawa Scale (Nos) For Assessing The Quality Of Nonrandomized Studies In Meta-Analysis. http://www.ohri.ca/programs/clinical_epidemiology/oxford web.ppt.

28 Higgins JP, Thompson SG, Deeks JJ, Altman DG: Measuring inconsistency in meta-analyses. BMJ 2003;327:557-560.

29 Steinhauser S, Schumacher M, Rucker G: Modelling multiple thresholds in meta-analysis of diagnostic test accuracy studies. Bmc Med Res Methodol 2016;16:97.

-30 Rucker G, Schumacher M: Summary ROC curve based on a weighted Youden index for selecting an optimal cutpoint in meta-analysis of diagnostic accuracy. Stat Med 2010;29:3069-3078.

-31 Yan S, Liu S, Zhao Y, Zhang W, Sun X, Li J, Jiang F, Ju J, Lang N, Zhang Y, Zhou W, Li Q: Diagnostic accuracy of HbA1c in diabetes between Eastern and Western. Eur J Clin Invest 2013;43:716-726.

-32 Markl B, Wieberneit J, Kretsinger H, Mayr P, Anthuber M, Arnholdt HM, Schenkirsch G: Number of intratumoral t lymphocytes is associated with lymph node size, lymph node harvest, and outcome in NodeNegative colon cancer. Am J Clin Pathol 2016;145:826-836.

-33 Noble F, Mellows T, McCormick ML, Bateman AC, Harris S, Underwood TJ, Byrne JP, Bailey IS, Sharland DM, Kelly JJ, Primrose JN, Sahota SS, Bateman AR, Thomas GJ, Ottensmeier CH: Tumour infiltrating lymphocytes correlate with improved survival in patients with oesophageal adenocarcinoma. Cancer Immunol Immunother 2016;65:651-662.

-34 Yodying H, Matsuda A, Miyashita M, Matsumoto S, Sakurazawa N, Yamada M, Uchida E: Prognostic significance of Neutrophil-to-Lymphocyte ratio and Platelet-to-Lymphocyte ratio in oncologic outcomes of esophageal cancer: A systematic review and meta-analysis. Ann Surg Oncol 2016;23:646-654.

- 35 Xue TC, Jia QA, Ge NL, Chen Y, Zhang BH, Ye SL: Imbalance in systemic inflammation and immune response following transarterial chemoembolization potentially increases metastatic risk in huge hepatocellular carcinoma. Tumour Biol 2015;36:8797-8803.

-36 Gu X, Gao X, Li X, Qi X, Ma M, Qin S, Yu H, Sun S, Zhou D, Wang W: Prognostic significance of neutrophil-tolymphocyte ratio in prostate cancer: Evidence from 16,266 patients. Sci Rep 2016;6:22089. 